hep-th/0612181

IPM/P-2006/065

IC/2006/132

\title{
Non-Abelian Magnetized Blackholes and Unstable Attractors
}

\author{
A.E. Mosaffa ${ }^{1}$, S. Randjbar-Daemi ${ }^{2}$, M. M. Sheikh-Jabbari ${ }^{1}$ \\ ${ }^{1}$ Institute for Studies in Theoretical Physics and Mathematics (IPM) \\ P.O.Box 19395-5531, Tehran, IRAN \\ E-mails: mosaffa, jabbari@theory.ipm.ac.ir \\ 2 The Abdus Salam International Centre for Theoretical Physics, \\ Strada Costiera 11 34014, Trieste, Italy \\ E-mail: seif@ictp.trieste.it
}

\begin{abstract}
Fluctuations of non-Abelian gauge fields in a background magnetic flux contain tachyonic modes and hence the background is unstable. We extend these results to the cases where the background flux is coupled to Einstein gravity and show that the corresponding spherically symmetric geometries, which in the absence of a cosmological constant are of the form of Reissner-Nordström blackholes or the $A d S_{2} \times S^{2}$, are also unstable. We discuss the relevance of these instabilities to several places in string theory including various string compactifications and the attractor mechanism. Our results for the latter imply that the attractor mechanism shown to work for the extremal Abelian charged blackholes, cannot be applied in a straightforward way to the extremal non-Abelian colored blackholes.
\end{abstract}




\section{Introduction and Motivation}

Stability analysis of systems of charged particles coupled to Einstein gravity in four dimensions is an old and well studied subject. Static, spherically symmetric solution with electric and/or magnetic charge, the Reissner-Nordström blackhole, has been shown to be stable under classical stability test. Charged or neutral scalars can also be added to the EinsteinMaxwell theory. A special case of the latter is the Einstein-Maxwell-Dilaton (EMD) theory. String theory toroidal compactifications at generic points in their moduli space indeed lead to a EMD theory with several $U(1)$ 's as well as dilatonic fields (e.g. see [1]). All these generalized charged blackholes are stable.

As the next generalization, one may couple non-Abelian gauge theories to Einstein gravity. These theories are hence called Einstein-Yang-Mills (EYM) theories [2]. Such theories have also been studied in some detail and it has been shown that they have an infinite discrete family of globally regular solutions as well as blackhole solutions for any given value of the horizon radius [3, 4, 5]. Despite having time independent colored globally regular or colored blackholes solutions, it has been argued that all of these solutions are unstable. (For a more detailed review see [6].)

When Dilaton or Higgs scalars are added to the system we obtain, respectively, EYMD or EYMH theories. These are the kind of theories which can arise from string theory compactifications, though not in generic points of the moduli space of toroidal compactifications. They also arise from compactification on group manifolds or generic Calabi-Yau manifolds. As we are interested in gravity theories arising from string compactifications, in this note we shall focus on these theories and review some of the results regarding the stability of such charged blackholes in section 2.2. We extend the results on the instability of the colored blackholes to a wider class in which the unstable (tachyonic) mode is not an $s$-wave.

In the rest of this note, we will first review the results of [7, 8], stating the existence of the tachyonic modes in the fluctuations of the Yang-Mills fields in a monopole background. In section 2.1, we complete the existing literature by showing that indeed the existence of these tachyonic modes leads to the instability of the background. In section 2.2, we review arguments in the GR literature in which it has been shown that a similar class of systems, namely the colored blackhole systems, are unstable. In section 3, we focus on the instabilities in the presence of gravitational field. In this section we show that if a Yang-Mills configuration has destabilizing modes among its fluctuations already in the flat space, then coupling to gravity will not change the situation as regards to stability. To this end first we show that the Dirac monopole in a $\operatorname{EYM}(\mathrm{D})$ theory generically has fluctuations which are localized in space but grow exponentially in time. With a given monopole configuration or 
the corresponding magnetic flux, the space time geometry is either of the form of (colored) Reissner-Nordström $(\mathrm{RN})$ blackhole or $A d S_{2} \times S^{2}$. We show that both of the two cases are suffering from a similar kind of instability present in the non-gravitational case. The solutions with minimal monopole charge are stable. This seems to have been overlooked in previous analysis.

For the blackhole solutions, our results extend the known instability arguments of the GR literature to a larger class, and in particular to the cases different from $s$-wave perturbations and monopole charges different from minimal value for the tensorial perturbations. The $A d S_{2} \times S^{2}$ is of relevance to the attractor mechanism which is at work for extremal blackhole

solutions in the Einstein-Maxwell-Dilaton (EMD) theories (e.g. see [9, 10]). The instability of the (extremal) colored blackholes and its near horizon geometry, the $A d S_{2} \times S^{2}$ background, implies that, if applicable at all, the attractor mechanism in its present form does not work for the colored non-Abelian blackholes. In section 4, we discuss that the EYMD or EYMH theories are very relevant to string theory and therefore the results of the colored blackhole instabilities apply to string theory compactifications. We end with concluding remarks and some interesting open problems.

\section{Review of instabilities in background non-Abelian magnetic flux}

This section is mainly a review of known results. In sections 2.1 and 2.2, respectively, we consider the non-gravitational and gravitational cases and point out that in both cases the fluctuations of non-Abelian gauge fields about a monopole background contain unstable modes.

\subsection{Linearized analysis of the gauge fields fluctuations}

Fluctuations of a non-Abelian Yang-Mills field around a magnetic monopole background on $R^{3+1}$ which has a non-vanishing flux through $S^{2}$, has tachyonic modes which can create instabilities [7]. To study such fluctuations it is sufficient to study the linearized Yang-Mills equations $D_{M} F^{M N}=0$, where $F^{M N}$ is the gauge field strength, around the solution of interest. To proceed we use the notation $M=\mu, m$, where $\mu$ ranges over $x^{0}=t$ and $x^{1}=r$ and $m$ ranges over $\theta$ and $\phi$. We also take the four dimensional metric of the form

$$
d s^{2}=g_{\mu \nu} d x^{\mu} d x^{\nu}+r^{2} g_{m n} d y^{m} d y^{n}
$$

where $g_{m n}$ denotes the standard metric on a $S^{2}$ of unit radius. Next we write

$$
W_{M}=\bar{A}_{M}+V_{M}
$$


where $W_{M}$ is the gauge field potential and $\bar{A}_{M}$ is the background potential corresponding to a Dirac monopole located at the origin (and hence has spherical symmetry)

$$
\bar{A}=\frac{1}{2} n^{i} T_{i}(\cos \theta-1) d \phi
$$

where $T_{i}$ is a basis of matrices in the Cartan sub-algebra of the Lie algebra of $G$ and, in an appropriate normalization, $n_{i}$ which correspond to the charge(s) of the monopole are integers. Yang-Mills equations, up to linear order in $V_{n}$, give,

$$
\nabla_{\mu}^{2} V^{n}+\frac{1}{r^{2}} D_{m}^{2} V^{n}-\left[D_{m}, D_{n}\right] V^{m}-\frac{i}{r^{2}}\left[V_{m}, \bar{F}^{m n}\right]=0 .
$$

In the above the covariant derivatives $\nabla^{\mu}$ and $\nabla^{m}$ are respectively constructed using the metrics $g_{\mu \nu}$ and $g_{m n}$ and the $m, n$ and $\mu, \nu$ indices are raised and lowered by metrics $g_{m n}$ and $g_{\mu \nu} . D_{m}$ is defined by

$$
D_{m} V_{n}=\nabla_{m} V_{n}-i\left[\bar{A}_{m}, V_{n}\right]
$$

and

$$
\bar{F}=-\frac{n_{i}}{2} T^{i} \sin \theta d \theta \wedge d \phi
$$

The above equations of motion are written in the Lorentz gauge

$$
\nabla_{\mu} V^{\mu}+\frac{1}{r^{2}} D_{m} V^{m}=0
$$

Our interest is primarily in the equation for $V^{n}$. We can express the commutators in terms of the curvature tensor. The equation simplifies to

$$
\nabla^{2} V^{n}+\frac{1}{r^{2}}\left\{\left(D_{m}^{2}-1\right) V^{n}-2 i\left[V_{m}, \bar{F}^{m n}\right]\right\}=0 .
$$

Expanding $V^{n}(t, r, \theta, \phi)$ in harmonics on $S^{2}$ yields an infinite number of fields in the $1+1$ dimensional space spanned by $t$ and $r$. The general formalism has been given in [14]. To perform such an expansion it is necessary to use complex basis in the tangent space of $S^{2}$ and denote the component of $V_{n}$ with respect to such basis by $V_{+}$and $V_{-}$. The equations then separate and we obtain

$$
\nabla^{2} V_{+}+\frac{1}{r^{2}}\left\{\left(D_{m}^{2}-1\right) V_{+}-2 i\left[V_{+}, \bar{F}_{-+}\right]\right\}=0
$$

Writing

$$
V_{+}=V_{+}^{i} T_{i}+V_{+}^{a} T_{a}
$$

where $T_{i}, T_{a}$ are, respectively, the generators in and outside the Cartan subalgebra satisfying

$$
\left[T_{i}, T_{a}\right]=\alpha_{i a} T_{a}
$$


the equation for $V_{+a}$ becomes

$$
\nabla^{2} V_{+a}+\frac{1}{r^{2}}\left\{\left(D_{m}^{2}-1\right) V_{+a}+n^{i} \alpha_{i a} V_{+a}\right\}=0 .
$$

This equation can be written in the more suggestive form of

$$
\nabla^{2} V_{+a}-\frac{1}{r^{2}} M^{2} V_{+a}=0
$$

where the "mass operator" $M^{2}$ is defined by

$$
M^{2}=-\left\{\left(D_{m}^{2}-1\right)+n^{i} \alpha_{i a}\right\}
$$

The spectrum of this operator is known. We know that for almost all non-Abelian $G$ 's it has a single negative eigenvalue (generically corresponding to degenerate eigenstates) and an infinite number of positive eigenvalues [7, 8]. The multiplicity of the mode with negative $M^{2}$, tachyonic modes 1 , is given by the dimension of the representation of the overall unbroken group to which they belong. For example, assume that the gauge group is $S U(2)$. The background gauge field is that of a monopole of charge $n$ which we take it to be a positive integer greater than one in the direction of $T_{3}$. The reason we exclude $n=1$ is that there are no negative $M^{2}$ modes for this value of $n$. In this case all the tangential fluctuations of the gauge field are spinorial and the Abelian embedding is stable under small fluctuations.

In this example, if there are no other symmetry breaking elements (like charged scalar fields or the Higgs fields) the unbroken part of the symmetry group in the $t, r$ space will be $U(1) \times S U(2)_{K K}$, where, $U(1)$ is the unbroken subgroup of the gauge group $S U(2)$ and the factor $S U(2)_{K K}$, is due to the spherical symmetry of the background solution. The tachyons in that case are charged under the $U(1)$ and belong to the spin $j=n / 2-1$ representation of $S U(2)_{K K}$. This in particular means that for even $n$ the tachyons are in tensorial representations of the rotation $S U(2)_{K K}$. Note that for odd $n$ the tachyons are spinorial objects with respect to $S U(2)_{K K}$. The $s$-wave tachyon corresponds to $n=2$. Moreover there are of $2 j+1=n-1$ complex tachyons (for $n>1$ ) with $M^{2}=-n / 2$ (for the tachyonic modes $\left.D_{m}^{2}=-j[7]\right)$.

One should, however, note that having negative $M^{2}$ is not in itself sufficient to conclude the instability of the field configuration, one needs also to analyze the propagation of these negative $M^{2}$ modes in the $t, r$ space.

\footnotetext{
${ }^{1}$ Note that if the 2 -dimensional metric is flat, i.e. $g_{\mu \nu}=\eta_{\mu \nu}$, and the 4-dimensional space-time is a product of $R^{2} \times S^{2}$ then $M^{2}$ will be precisely the mass ${ }^{2}$ operator in $R^{2}$ and the appellation of tachyonic mode will be exact. By slight misuse of language we shall continue to call $M^{2}$ the mass operator even for a non flat $g_{\mu \nu}$ and for non factorized geometries.
} 
If we assume $g_{\mu \nu}=\eta_{\mu \nu}$, the fluctuation equation (2.13) reduces to the simple wave equation

$$
-\partial_{t}^{2} V_{+}+\partial_{r}^{2} V_{+}-\frac{1}{r^{2}} M^{2} V_{+}=0
$$

The most general solution to this equation turns out to be

$$
V_{+}=e^{i E t} e^{i E r} r^{\beta}\left(C_{1} F(\beta, 2 \beta ;-2 i E r)+C_{2}(-2 i E r)^{1-2 \beta} F(1-\beta, 2-2 \beta ;-2 i E r)\right.
$$

where $F(a, c ; x)$ is a confluent hypergeometric function and $C_{1}$ and $C_{2}$ are the two integration constants. $\beta$ is a solution of

$$
(\beta-1 / 2)^{2}=M^{2}+1 / 4 \text {. }
$$

For $M^{2}<-1 / 4$, which is the case of interest for us, the right hand side of this equation can be negative yielding a complex solution for $\beta$. One can show that in this case there is a choice of the integration constants which allow a normalizable solution with a pure imaginary $E$. Such a solution will be localized in space but delocalized (with exponential growth) in time and will signal an instability.

If there are other charged fields in the model they may contribute to the tachyonic mass and lift it to non-negative values. One such possibility is adding the Higgs field in the adjoint (the Georgi-Glashow model) or the fundamental representations. For the former, there are singular or regular ('t Hooft-Polyakov) monopole solutions.

Let us consider the Georgi-Glashow model, with the group $S U(2)$ and a Higgs potential whose value vanishes in its minimum given by $\left|\phi_{v a c}\right|^{2}=v^{2}$ (e.g. see section 1.4 of [11]):

$$
\mathcal{L}=-\frac{1}{4} \sum_{i=1}^{3}\left(F_{M N}^{i} F^{i M N}+\frac{1}{2} D_{M} \phi^{i} D^{M} \phi^{i}\right)-\frac{\lambda}{4}\left(\phi^{2}-v^{2}\right)^{2}
$$

where

$$
\begin{aligned}
F_{M N}^{i} & =\partial_{[M} W_{N]}^{i}-g_{Y M} \epsilon^{i j k} W_{M}^{j} W_{N}^{k} \\
D_{M} \phi^{i} & =\partial_{M} \phi^{i}-g_{Y M} \epsilon^{i j k} W_{M}^{j} \phi^{k} .
\end{aligned}
$$

Our arguments in an obvious way generalizes to a generic non-Abelian gauge group $G$. The singular monopole solution is given by (see eq.(23) of [11])

$$
\begin{gathered}
\vec{\phi}_{v a c} \cdot \vec{\phi}_{v a c}=v^{2}, \\
\vec{W}_{M}=\frac{1}{g v^{2}} \vec{\phi}_{v a c} \times \partial_{M} \vec{\phi}_{v a c}+\frac{1}{v} \vec{\phi}_{v a c} A_{M}^{0}
\end{gathered}
$$

where the arrows denote the (adjoint) gauge indices and $A_{M}^{0}$ is an arbitrary vector, and singlet of the gauge group. With the above it is easy to check that $\partial_{M} \vec{\phi}_{v a c}+i g \vec{W}_{M} \times \vec{\phi}_{v a c}=0$. The "magnetic" monopole $U(1)$ gauge field strength is then given by $\bar{F}_{M N}=\frac{1}{v} \vec{\phi}_{v a c} \cdot F_{M N}$. This 
monopole solution is obviously singular. We choose the three components of $\vec{\phi}$ to be given by $v(\sin \theta \cos m \varphi, \sin \theta \sin m \varphi, \cos \theta), m \in \mathbb{Z}$.

The stability analysis in this case has been carried out in [12] for the $s$-wave perturbations (which corresponds to $m=1$ in this case). In fact the gauge transformation which brings $\phi$ to the direction of $z$-axis will map $W$ to our $\bar{A}$ of (2.3) with $m=2 n 2$ Repeating the analysis of the spectrum of gauge field fluctuations about the above when Higgs is turned on, will therefore produce the same result given by equation (2.13) but the $M^{2}$ is now replaced with

$$
M^{2}=-\left\{\left(D_{m}^{2}-1\right)+2 n\right\}+r^{2} g_{Y M}^{2} v^{2}
$$

where the last term is due to the Higgs giving mass to the gauge fields. It can be checked that for small enough $r, r \lesssim r_{m}$, with

$$
r_{m} \sim\left(g_{Y M} v\right)^{-1}
$$

the last term becomes subdominant and we again find the tachyonic instability.

Finally we consider the regular 't Hooft-Polyakov solution. The (approximate) form of solution may be found in eq.(29) of [11]. This solution at large $r$ reduces to (2.20). At $r \lesssim r_{m}$, however, the solution behaves differently and in particular at $r=0$ it is regular. In this case the monopole is an object of effective radius of $r_{m}$, rather than a point like object. The stability analysis for this case, due to the $r$-dependence of the background Higgs and the gauge fields becomes very messy, however, one expects this solution to be stable. This expectation is supported by topological reasons and, in our setting, by the observation that due to the monopole profile, the $M^{2}$ has a non-trivial $r$ dependence and unlike the previous case, does not change sign around $r_{m}$.

Similar arguments could be made for the fundamental Higgs case and when we have dyons.

\subsection{The gravitational setting, instability in EYM(D) theoreis}

In this section we give a brief review of the non-Abelian gravitating solutions of EYMD theory in four dimensions (for a more detailed review see [6] and references therein). These solutions can be either point like, globally regular, asymptotically flat (solitonic) or of the blackhole type. There are a number of no-go theorems assuring that stationary solitonic solutions do not exist in pure gravity or in YM theory. In gravity, this is referred to as the Lichnerovicz's theorem which follows from the fact that gravitational systems are purely

\footnotetext{
${ }^{2}$ It is known that with the adjoint Higgs one will only obtain Dirac monopoles of even charge. In particular the lowest possible charge in this setting corresponds to $n=2$ in the notations of (2.3). This is the reason that our $s$-wave is obtained for $n=2$.
} 
attractive and hence there is no way to make a balance of forces to keep the gravitational soliton stationary. On the other hand the YM system is purely repulsive and therefore it cannot support what is called a "classical glueball".

When combined together, gravity and YM can in principle make a balance between forces to support stationary neutral solitonid 3 [2] or neutral and charged blackhole solutions [4]. Similar class of solutions have also been constructed for systems including a dilatonic scalar field i.e. EYMD [5] 4$]$

In this section we will take the gauge group to be $S U(2)$ and consider four dimensional, spherically symmetric, asymptotically flat static solutions only. To summarize the results already discussed in the literature and introduce the terminology used, it is convenient to start with the most general ansatz for spherically symmetric solutions

$$
\begin{aligned}
d s^{2} & =\sigma^{2} N(d t+\alpha d r)^{2}-\frac{1}{N} d r^{2}-R^{2}\left(d \theta^{2}+\sin ^{2} \theta d \phi^{2}\right) \\
W & =a T_{3}+\operatorname{Im}\left(w T_{+}\right) d \theta-\frac{n}{2} R e\left(w T_{+}\right) \sin \theta d \phi+\frac{n}{2} T_{3} \cos \theta d \phi
\end{aligned}
$$

where $T_{i}(i=1 \cdots 3)$ are the $S U(2)$ generators and $T_{+}=T_{1}+i T_{2}$. Furthermore, $a$ is a real one form $a=a_{0} d t+a_{r} d r$ and the complex scalar $w$ as well as $\sigma, N, \alpha$ and $R$ only depend on $(t, r)$. The integer $n$ is the monopole charge.

A study of the equations of motion for the YM field indicates that for $n \neq 2$ one should necessarily have $w=0$. Solutions of this type are called embedded Abelian (eA), as the potential in this case can be written in terms of the $U(1)$ potential $\mathcal{A}=\operatorname{Tr}\left(W T_{3}\right)=a+$ $\frac{n}{2} \cos \theta d \phi$. The stationary (eA) solutions are hence, always charged and are blackholes, the so-called colored blackholes.

On the other hand, the non-Abelian (nA) solutions which can only be achieved when $n=2$, may be solitonic or blackholes, but always neutral. The stability of these (nA) solutions against spherically symmetric perturbations has been extensively studied in the literature with the overall result that these solutions are all unstable. There are two classes of the unstable, tachyonic, modes; those in which the gravity fluctuations are turned off, the topological or odd parity modes, and those which also involve metric perturbations, the gravitational or even parity modes.

The above system can be generalized to the one which is more relevant to string theory, by adding a dilaton field to the EYM to end up with the EYMD system. The dilaton, $\phi$, couples to the YM action in the form of $e^{2 \gamma \phi} L_{Y M}$ where $\gamma \geq 0$ is a parameter. For any given $\gamma$, the (nA) solutions of the EYMD system as well as their stability behavior are the same as

\footnotetext{
${ }^{3}$ There is a no-go theorem stating that there exists no charged soliton in EYM 13 .

${ }^{4}$ Systems including scalar fields in the non-trivial representation (fundamental or adjoint) of the gauge group, the Higgs field, have also been extensively studied under the name of EYMH [12, 15, 16, 17.
} 
those of EYM, i.e. there are both odd and even parity tachyonic modes [5, 18]. The extreme limits of $\gamma \rightarrow 0$ and $\gamma \rightarrow \infty$ respectively correspond to the EYM and YMD (in flat space). The latter which is essentially the case we analyzed in section 2.1 has the same features of EYM i.e. the dilaton, providing a purely attractive system, can replace gravity leading to the same sort of solutions. The EYMD will thus interpolate between these extreme systems and has the same features of the two.

Another system of interest is an EYM with a cosmological constant; EYM- $\Lambda$ [19]. The solutions of this system are asymptotically (A)dS for (negative) positive values of $\Lambda$. For $\Lambda>0$, the moduli space of $(\mathrm{nA})$ solutions is essentially that of the EYM system and all the solutions are still unstable, with the same tachyonic modes. For $\Lambda<0$, however, the situation is different. The main difference is that, the presence of negative $\Lambda$ allows for a new continuous family of non-Abelian charged solitonic or blackhole solutions. The stability analysis for this case shows that [20, 21], although the solutions are still generically unstable, we now have the possibility of having stable solutions which of course do not have any counterpart in the $\Lambda=0$ theory.

\section{Instability of EYM(D) solutions with magnetic flux}

In the previous section we reviewed the instability of the so-called non-Abelian solitonic or blackhole solutions. In this section we apply the analysis of section 2.1 to all possible spherically symmetric solutions to the EYM action

$$
S=\int d^{4} x \sqrt{-g}\left(\frac{1}{8 \pi G_{N}} R+\operatorname{Tr} F_{M N} F^{M N}\right)
$$

when the magnetic flux $\bar{F}_{m n}(2.6)$ is turned on. With the gauge fields of the form of (2.6) and noting that $\bar{F}$ only takes values in the Cartan subalgebra of the gauge group $G$, finding solutions of the field equations is essentially identical to the one in Einstein-Maxwell theory. These solutions, if asymptotically flat, are of the form of (magnetized) Reissner-Nordström $(\mathrm{RN})$ blackholes of arbitrary magnetic charges $n_{i}$. There is only one other class of solutions which are not asymptotically flat. These are non-singular and of the form of $A d S_{2} \times S^{2}$. The latter may be obtained as the near horizon geometry of the extremal RN solutions.

We show that turning on gravity does not remove the instabilities we discussed in section 2.1 and the same modes of the gauge field which were tachyonic in the non-gravitational case ${ }^{5}$ remain tachyonic. Hence, we are led to the conclusion that

\footnotetext{
${ }^{5}$ In the RN solutions generically there are four parameters (or quantum numbers) appearing, the Newton constant $G_{N}$, the Yang-Mills coupling $g_{Y M}$, and monopole charges $n_{i}$ and the ADM mass of the blackhole. By non-gravitational limit we mean $G_{N} \rightarrow 0$ keeping the other parameters fixed.
} 
the presence of gravity is irrelevant to the instability results of the non-gravitational case. Inclusion of dilatonic scalar fields does not alter this result. Addition of Higgs scalars, being a charged field under YM, can however push some or all of the tachyonic modes inside the horizon and thus may stabilize the system.

In section 3.1 we analyze the (in)stability of embedded Abelian, colored blackhole case. In comparison to the extensive literature on the non-Abelian solutions, the literature on the embedded Abelian case is slim and only the case with $s$-wave Gerogi-Glashow monopole has been considered in detail, showing that such perturbations lead to instabilities [12.6 In section 3.2 we study the instability of the $A d S_{2} \times S^{2}$ solution. In section 3.3 we consider EYMD theory and discuss the implication of the instability to the attractor mechanism.

\subsection{Instability of generic $4 d$ colored blackholes}

The (in)stability analysis of a colored, magnetized RN blackhole amounts to studying (2.13) with the proper choice for the metric $g_{\mu \nu}$. In order that we take the metric of the form

$$
d s^{2}=-f d t^{2}+f^{-1} d r^{2}+b^{2} d \Omega_{2}^{2}
$$

where $f$ and $b$ are functions of $r$. Equation (2.13) written with this metric yields

$$
-f^{-1} \partial_{t}^{2} V_{+}+\partial_{r}\left(f \partial_{r} V_{+}\right)-\frac{1}{b^{2}} M^{2} V_{+}=0
$$

where $M^{2}$ has the same expression as in (2.14). For concreteness, we take the gauge group to be $S U(2)$ for which the spectrum of $M^{2}$ has a simple form. To analyze the above equation, define the new coordinate $\rho$

$$
d \rho=f^{-1} d r
$$

In this new coordinate system the metric becomes $d s^{2}=f\left(-d t^{2}+d \rho^{2}\right)$. Replacing

$$
V_{+}=e^{i E t} \psi(\rho)
$$

equation (3.3) then takes the form of a Schrödinger equation

$$
-\partial_{\rho}^{2} \psi+U(\rho) \psi=E^{2} \psi
$$

with the energy $E^{2}$ and the potential $U(\rho)$

$$
U(\rho)=\frac{f}{b^{2}} M^{2}
$$

\footnotetext{
${ }^{6}$ Reference [12] also considers some tensorial non spherically symmetric modes. As discussed in section 2.1. however, for a generic monopole of charge $n$, e.g. in the $S U(2)$ gauge theory, the spin of the tachyonic mode is $n / 2-1$. Our discussion applies to modes belonging to any representation of the rotation $S U(2)$, tensorial as well as spinorial.
} 
The question of instability, like the flat space case, therefore reduces to the existence of normalizable, negative $E^{2}$ states (the bound states). The normalizability condition should now be imposed taking the non-flat background metric into account. That is,

$$
\int d \rho \sqrt{f} b^{2}|\psi|^{2}<\infty
$$

For the asymptotically flat case, like ours, at large $r$ (where the normalizability concerns may arise) the measure reduces to that of standard flat space $r^{2} d r$. This question could be answered once the function $f$ is specified. (One should also note that functions $f$ and $b$ should be written in terms of $\rho$.) In the cases of interest for us we are dealing with blackholes and hence the function $f(r)$ vanishes at the horizon and generically we have inner and outer horizons, respectively $r_{-}$and $r_{h}$. The Schrodinger equation should be analyzed for $r \geq r_{h}$. The wave-function $\psi$ should vanish for $r<r_{h}$, as the region inside the horizon is cut-off from the rest of the space.

As the first example let us consider the extremal Reissner-Nordström (RN) case, where

$$
f=\left(1-\frac{Q}{r}\right)^{2}, \quad b=r
$$

$Q$ and the monopole charge $n$ are related as

$$
Q=\frac{n}{2 g_{Y M}} \sqrt{4 \pi G_{N}} \equiv \frac{n}{2 g_{Y M}} M_{P l}^{-1} .
$$

In this case the explicit relation between $\rho$ and $r$ is

$$
\rho=r-Q+2 Q \ln \frac{r-Q}{Q}-\frac{Q^{2}}{r-Q}
$$

where, for convenience, we have chosen the integration constant such that $r=2 Q$ corresponds to $\rho=0$. Note that $\rho$ ranges from $-\infty$ to $+\infty$ as $r$ ranges from the horizon $r_{h}=Q$ to $+\infty$. This coordinate does not cover the region inside the horizon. The $(\rho, t)$ coordinate system is usually called the Regge-Wheeler coordinates.

It is easy to see that $\rho= \pm \infty$ are inflection points of the potential and that $U$ vanishes at these points. Furthermore, $U(\rho)$ has one more extremum at $\rho=0$ which is a minimum for negative $M^{2}$ and a maximum for positive $M^{2}$ and its value at this extremum is $\frac{M^{2}}{16 Q^{2}}$. $U(\rho)$ is smooth everywhere and $U(\rho)=U(-\rho)$. The case of interest for us is of course when $M^{2}$ is negative which is depicted in Fig.1.

Qualitatively our quantum mechanical problem is very similar to the motion of a particle in a potential of the form $\frac{M^{2}}{\cosh ^{2} \rho}$ (the latter has been discussed in [22]). This potential has a finite number of of normalizable discrete modes (bound states) for $\frac{M^{2}}{16 Q^{2}} \leq E^{2} \leq 0$. For $E^{2} \leq \frac{M^{2}}{16 Q^{2}}$ of course there will be no solutions. Existence of negative energy bound states, as 


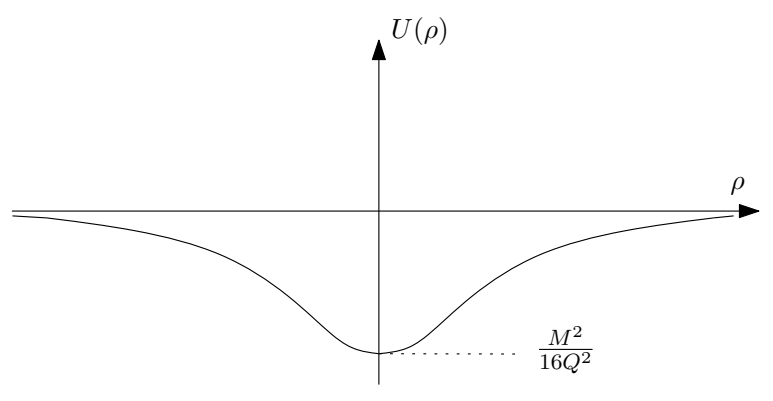

Figure 1: The Potential given in eq.(3.6) for the Extremal Reissner-Nordström blackhole, with $M^{2}=-n / 2$. This potential has negative energy bound states.

discussed earlier, means that the unstable modes, i.e. the modes which grow exponentially in time, which were also present in the flat background survive even when the back reaction of the magnetic monopole on geometry is taken into account.

One may repeat the above analysis for more general non-extremal cases where

$$
f(r)=\frac{1}{r^{2}}\left(r-r_{-}\right)\left(r-r_{h}\right)
$$

The qualitative behavior of the potential is essentially the same as the extremal case. That is, for negative $M^{2}$ the potential $U(\rho)$ has a minimum outside the horizon, and it is always negative. Furthermore, in the $\rho \rightarrow \pm \infty, U(\rho)$ asymptotes to zero.

Let us now add the non-singlet scalar field, the Higgs field. As discussed in section 2.1, this is done by replacing the $M^{2}$ by $M^{2}+g_{Y M}^{2} v^{2} b^{2}$ (cf. eq.(2.21)). $U(\rho)$ becomes,

$$
U(\rho)=\frac{\left(r-r_{-}\right)\left(r-r_{h}\right)}{r^{2}}\left(g_{Y M}^{2} v^{2}+\frac{M^{2}}{r^{2}}\right)
$$

where $v$ is the Higgs expectation value and $\rho$ and $r$ are related as in (3.10). The potential $U(\rho)$, unlike the previous case does not necessarily have bound states. As the expectation value of the Higgs field increases, the minimum of $U(\rho)$ is pushed behind the horizon. This can eventually result in a potential without any bound states and hence large Higgs vev can remove the instability 7 . Intuitively and as a rough measure, when we add Higgs there are two length scales in the problem, one is the horizon size $r_{h}$ and the other is the monopole size $r_{m}$ (2.22). When $r_{h}>r_{m}$ the monopole is completely sitting behind the horizon and hence the instability analysis is similarly to a "point" charge. In this case, although the potential has a minimum, its minimum is sitting behind the horizon and hence do not form a bound state. Therefore, we do not see the instability. When $r_{m}>r_{h}$ we have a smoothed out,

\footnotetext{
${ }^{7}$ This observation had already been made in [12] for the $s$-wave instabilities.
} 
extended monopole. As has been depicted in Fig,2 in this case the potential does have the possibility of bound state, and hence the instability. The same qualitative features remain when we consider non-extremal case with the Higgs. The above potential generalizes the results of [12] to the case with arbitrary monopole charge.
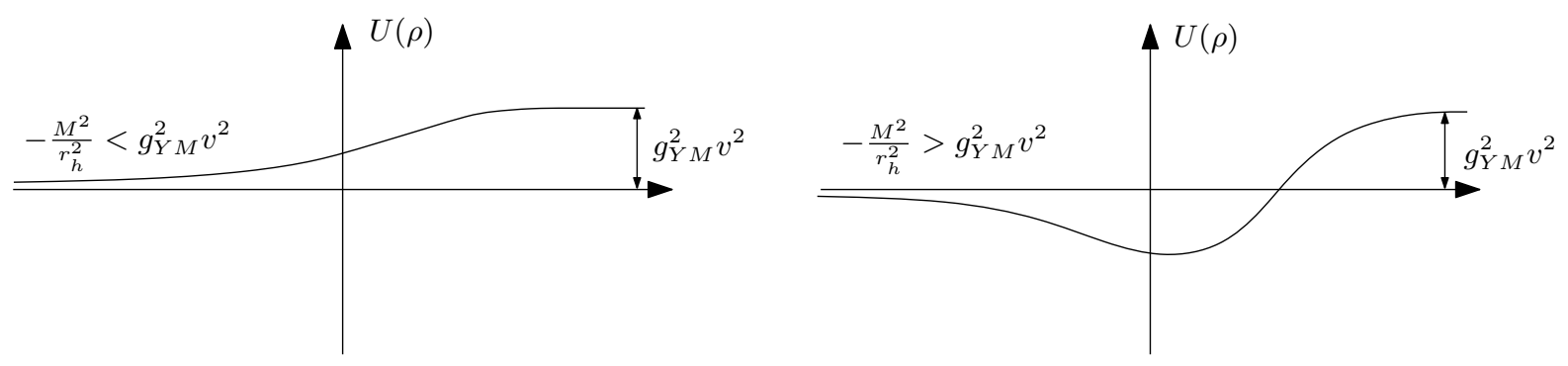

Figure 2: The potential for generic RN blackhole with the Higgs. As we increase the Higgs expectation value $v$ the minimum of the potential is pushed to the left and eventually can be removed. The Figure on the left show the case with a large Higgs and the right one shows the case with small $v$.

It turns out that bound states and hence unstable modes appear if $M^{2}<0$ and if

$$
\frac{g_{Y M}}{2 \pi} M_{b h}<\frac{c \sqrt{-M^{2}} M_{p}^{2}}{v}+\frac{v}{c \sqrt{-M^{2}}} \frac{n^{2}}{4}
$$

where $M_{p}$ is the planck mass and $M_{b h}=\frac{r_{-}+r_{h}}{2 G}$ is the ADM mass of the blackhole. $c$ is a positive number smaller than one and arises from the details of quantum mechanics analysis of having a bound state. For the extremal case, $M_{b h} G=Q, c$ approaches one.

\subsection{Instability in $A d S_{2} \times S^{2}$ background}

In this part we consider the other class of solutions to the EYM theory in which the field strength is given by (2.6), the $A d S_{2} \times S^{2}$. This solution can be obtained as the near horizon geometry of extremal colored blackholes and hence this case is of particular interest because of its possible relevance to the attractor mechanism [23, 24]. It has been discussed that the instability in such systems is completely determined by the large distance behavior of the radial equation [15]. In this section we show that the instability we have discussed in the previous sections is also seen in the near horizon geometry.

It is straightforward to check that the $A d S_{2} \times S^{2}$ :

$$
d s^{2}=R_{1}^{2}\left(-f(r) d t^{2}+f^{-1} d r^{2}\right)+R_{2}^{2} d \Omega_{2}^{2}
$$

where $R_{1}$ and $R_{2}$ are respectively radii of the $A d S_{2}$ and $S^{2}$ parts, and $f(r)$ is

$$
\begin{array}{ll}
f(r)=r^{2} & \text { for the Poincare }{ }^{\prime} \text { patch } \\
f(r)=1+r^{2} & \text { in the global } A d S \text { coordinates }
\end{array}
$$


when $R_{1}=R_{2}=Q$ is a solution to our EYM theory. This solution can be obtained in the near horizon limit of the four dimensional extremal Reissner-Nordström blackhole of previous section. Although for our case, the EYM theory and when four dimensional cosmological constant is zero, always $R_{1}=R_{2}$, in our analysis we keep the $A d S$ and the sphere radii arbitrary.

Our instability analysis goes through the same as before. We end up with looking for the possible bound states of the Schrödinger equation (3.5), in which the potential is given by (3.6) with $b$ replaced by $R_{2}$. That is, 8

$$
U(\rho)=\left\{\begin{array}{cc}
\frac{R_{1}^{2}}{R_{2}^{2}} M^{2} \frac{1}{\rho^{2}} & -\infty<\rho<+\infty \\
\frac{R_{1}^{2}}{R_{2}^{2}} M^{2} \frac{1}{\cos ^{2} \rho} & -\frac{\pi}{2}<\rho<+\frac{\pi}{2} \quad \text { in the global AdS coordinates }
\end{array}\right.
$$

To explore the bound states and discuss the instability we need to impose the correct normalizability and boundary conditions. In the case where we have a causal boundary, like the $A d S_{2}$ case, the suitable boundary condition for the wave function is that of a particle inside a box, with the walls of the box at the position of the boundary, i.e. $\rho=0$ in the Poincare' patch and $\rho= \pm \pi / 2$ for the global $A d S_{2}$. The form of the potential for negative $M^{2}$ is depicted in Fig 3 , The normalization conditions read

$$
\int_{0}^{\infty} d \rho \frac{1}{\rho}|\psi|^{2}<\infty \quad \text { for the Poincare' patch }
$$

and

$$
\int_{-\pi / 2}^{\pi / 2} d \rho \frac{1}{\cos \rho}|\psi|^{2}<\infty \quad \text { in the global } A d S \text { coordinates. }
$$

The above Schrödinger equation is in fact exactly the same as the wave equation for a massive scalar field of mass squared $M_{0}^{2}=\frac{R_{1}^{2}}{R_{2}^{2}} M^{2}$ in the $A d S_{2}$. Normalizable negative energy states exist if $M_{0}^{2}<-1 / 4$. To see this, let us for example consider the Schrödinger equation for the Poincare' coordinates and focus on the equation around $\rho=0$. The solutions can be of the form $\psi \sim \rho^{\beta}$, where $\beta$ satisfies (3.3) with $M^{2}$ replaced by $M_{0}^{2}$. As in the flat space case in order to have instability, $\beta$ should be a complex number. The above discussion results in the well-known fact that, to have tachyonic instability coming from a minimally coupled scalar in an $A d S$ background, we need to violate the Breitenlohner-Freedman bound [26], which for the $A d S_{2}$ case is exactly $M_{0}^{2}<-1 / 4$. For the case when the four dimensional cosmological constant vanishes, $R_{1}=R_{2}$ and hence $M_{0}^{2}=M^{2}$. If we choose our non-Abelian

\footnotetext{
${ }^{8}$ Note that the $A d S_{2}$ case is special in the sense that it has two disconnected one dimensional boundaries [25]. In our case this means that the range of $\rho$ coordinate can be extended to the full range of $(-\infty,+\infty)$ in the Poincare' patch, and to $\left(-\frac{\pi}{2},+\frac{\pi}{2}\right)$ in the global $A d S_{2}$. The boundaries are then located at $\rho=0$ for the former and at $\rho= \pm \frac{\pi}{2}$ for the latter.
} 

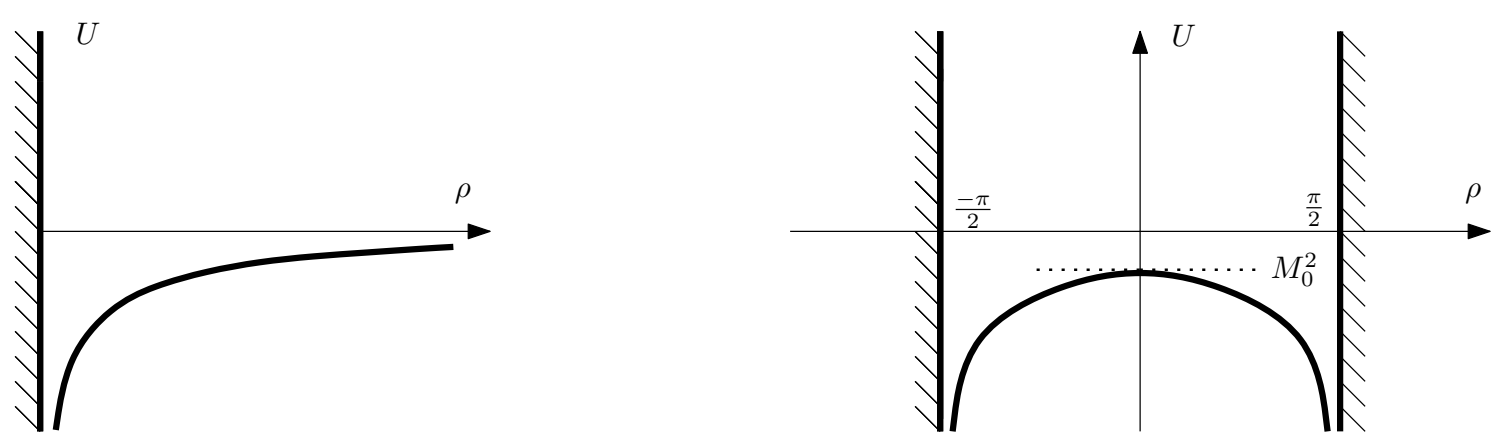

Figure 3: The potentials for the $A d S_{2}$ case when $M_{0}^{2}=\frac{R_{1}^{2}}{R_{2}^{2}} M^{2}$ is negative. The left figure corresponds to the Poincare' patch (as well as to the flat space) and the one on the right corresponds to the global coordinate.

gauge group to be $S U(2)$, with a monopole charge $n(n>1), M_{0}^{2}=-n / 2$ (cf. discussions of section 2.1) and hence we have tachyonic instability.

Here we briefly address the case $R_{1} \neq R_{2}$. This happens when we study near horizon limit of an extremal charged blackhole in a background of non-vanishing four dimensional cosmological constant $\Lambda$ [9, 28]. We then have

$$
R_{2}^{-2}-R_{1}^{-2}=2 \Lambda, \quad Q^{2}=R_{2}^{2}\left(1-R_{2}^{2} \Lambda\right)
$$

where $Q$ and the monopole charge $n$ are related as in (3.9). Therefore, by tuning up $|\Lambda|$ we can increase the $R_{2} / R_{1}$ ratio. When $\frac{R_{1}^{2}}{R_{2}^{2}}$ ratio exceeds $1 / 2 n$ we do satisfy the BreitenlohnerFreedman bound and hence there is no tachyon instability. More detailed and exhaustive analysis of this case, when a non-zero cosmological constant is turned on, is postponed to future works [27].

It is readily seen that for both cases we do have bound states and hence the persistence of the instability. It is remarkable that in the Poincare' patch the potential in terms of $\rho$ has exactly the same expression as the similar case in the flat space ( $c f$. equation (3.3)). Note also that, it is $A d S$ in the Poincare patch that is obtained as the direct result of taking the near horizon limit. This may give a direct realization of the observation we made in the beginning of this section.

As another remark we would like to note that in taking the near horizon limit we are replacing the localized charges with fluxes (see e.g. [28]). This in particular implies that the instability result is not limited to the charges and "monopole" configuration, but is a generic feature for the cases we are dealing with non-vanishing non-Abelian fluxes. 


\subsection{Instability in EYMD and implications for the attractors}

So far we have mainly focused on the EYM theory with a simple gauge group $G$. We also discussed the EYMH theory and argued how addition of a Higgs field can change the (in)stability of the background magnetic flux. Here we would like to address implications of addition of the singlet scalar fields (the dilatons) to EYM. This is the case of relevance to the attractor mechanism [10]. The attractor mechanism simply states that the near horizon geometry of the extremal charged blackholes only depends on the charges and is independent of the other details of the theory, and in particular the moduli, if present [10]. In other words, there are (infinitely) many extremal blackhole solutions which reduce to the same solution in the near horizon geometry and that the dynamics on the horizon is decoupled from the dynamics of the rest of the space [23]. This result has been used to study the entropy of the extremal blackholes [28] and to identify the micro-state counting of these blackholes [29].

For the cases where we have a single dilaton field and a simple gauge group $G$, presence of the dilaton field does not essentially change the equations of motion for the gauge fields and following the line of computations we have presented here, one can readily show that the same mode of the gauge field fluctuation which we have shown to be tachyonic remains tachyonic in the spherically symmetric magnetized solutions of the EYMD theory.

In order to study the implications for the attractor mechanism, recalling that when only the magnetic charges (fluxes) are turned on one needs to have more than one $U(1)$ factors (e.g. see [10]), we consider a more general case of the EYMD theory where our gauge group is a product of several simple factors and also several dilaton fields are present:

$$
S=\int d^{4} x \sqrt{-g}\left(\frac{1}{8 \pi G_{N}} R+\operatorname{Tr} F_{M N}^{a} F^{b M N} f_{a b}(\phi)-g(\phi)_{i j} \partial_{M} \phi^{i} \partial^{N} \phi^{j}\right)
$$

where $a, b$ run over $1, \cdots, k$ ( $k$ is the number of simple factors in the generic gauge group $G), \phi^{i}$ are scalar dilaton fields and the range of $i, j$ could be arbitrary, independent of $k$, $g_{i j}$ is the metric on the moduli space of the dilaton fields. Gauge invariance implies that $f_{a b}(\phi)=e^{\alpha_{a i} \phi^{i}} \delta_{a b}$.

The extremal solutions of the above EYMD theory are of relevance to the attractor mechanism [10]. For concreteness let us choose the gauge group to be $S U(2) \times S U(2)$ (i.e. $k=2$ ) and take a model with a single dilaton and $f_{a b}(\phi)=\delta_{a b} e^{\alpha_{a} \phi}, a, b=1,2$. The exact attractor solution of reference [10] can be readily embedded in this model. As shown in this reference for $\alpha_{1} \alpha_{2}<0$ there exits an exact attractor solution. Assuming $\phi=$ const, the monopole and blackhole configuration of previous section will correspond to the zeroth order solution of [10]. It is clear that the analysis of sections 2.4.2 to 2.4.4 of this reference can be carried through verbatim. The instability in non-Abelian flux backgrounds, however, still remains there, when the background charge is different from $n=1$ for each $S U(2)$. 
It is also straightforward, along the line of section 3.2 , to check that the $A d S_{2} \times S^{2}$ type solutions to the above EYMD theory are also unstable. In an obvious way one can show that the instability remains for (the extremal) solutions to the above EYMD (3.16) in its most general form.

As we discussed, the solutions to the EYMD theories which show the attractor behavior, independently of the value of the dilaton fields at infinity and the moduli, all suffer from the tachyonic instability and the runaway behavior in the small fluctuations of some of the components of the gauge fields. Thus except for the values of the magnetic charge $n=1$ for each $S U(2)$ factor, before studying the attractor property, it is therefore necessary to address the instability issue first.

\section{Relevance of colored blackholes to string theory}

We have discussed that EYMD theories have unstable regular or blackhole solutions. The instability manifests itself in the modes with imaginary frequency. The EYM and YMD theories are two specific limits of the EYMD theory. In this part we discuss the relevance of EYMD theories to string theory.

\subsection{Non-Abelian gauge theories and Calabi-Yau compactifications}

Let us start with standard $10 d$ superstrings and first consider the heterotic strings compactified down to $4 d$. The low energy effective theory obtained in this way is generically a $4 d$ (super)gravity theory plus some scalars (dilatons) and some gauge fields. In the toroidal compactification, we start with $E_{8} \times E_{8}$ or $S O(32) 10 d$ heterotic strings and end up with $\mathcal{N}=4$ supergravity in $4 d$ with gauge group which is a subgroup of $E_{8} \times E_{8}$ or $S O(32)$, plus 12 other $U(1)$ vector fields coming from the ten dimensional metric and two form $B$-field upon KK reduction 9 . In a generic point of the moduli space, where all the $16 E_{8} \times E_{8}$ or $S O(32)$ Wilson line moduli are turned on, the gauge group is Higgsed down to $U(1)^{16}$ [30]. Similarly, if we start with type I, type II or M theories, upon toroidal compactification to $4 d$, generally we obtain a $4 d$ supergravity with $U(1)^{28}$ gauge group. Therefore, at generic points of moduli spaces of string/M theory toroidal compactifications non-Abelian gauge groups may be completely broken, and hence the instability discussed here, does not show up 10 At

\footnotetext{
${ }^{9}$ It is possible that in the self T-dual points of the compactification moduli space this Abelian $U(1)^{12}$ enhances to non-Abelian $S U(2)^{12}$ or other larger groups of rank 12 [30].

${ }^{10} \mathrm{It}$ is worth noting that closed strings on a torus, when a background magnetic flux larger than some critical value is turned on, contain tachyonic modes [31. It has been argued that this tachyonic instability can signal a phase transition in the system [31.
} 
non-generic points, where some of the Wilson line moduli are not turned on, we do obtain non-Abelian gauge groups.

The toroidal compactification, although the simplest, is not the most interesting string compactification. A phenomenologically viable string compactification should lead to nonAbelian GUTs or the standard model. For this purpose the Calabi-Yau (CY) compactification is a better choice. In this case, for the heterotic or type I case, in the non-generic points of the compactification moduli space, we obtain non-Abelian gauge groups which are subgroups of $E_{8} \times E_{8}$ or $S O(32)$. In these cases we again face the generic instability of the colored blackholes we discussed here. Even if we are only interested in classical configurations which are free of these instabilities, the fact that these states exist among the classical solutions of the theory, at quantum level, can induce instability.

\subsection{Non-Abelian gauge theories in G/H compactifications}

The other class of compactifications which leads to non-Abelian gauged supergravities in $4 d$ are compactification on non-Ricci-flat manifolds, e.g. $G / H$ coset manifolds, $G$ being a compact group. In these cases the isometries of the compactification manifold, $G$, appears as the gauge group in the lower dimensional theory. Moreover, generically we also obtain a non-vanishing cosmological constant in the lower dimensional theory. The value of the cosmological constant is proportional to the scalar curvature of the compactification manifold, though with the opposite sign. Hence, in these cases we are dealing with (supersymmetric) EYMD- $\Lambda$ theory. As discussed in section 2.2, appearance of $\Lambda$ does not generically cure the instability of non-Abelian solutions. There is, however, the possibility of having stable nonAbelian solutions. The EYMD- $\Lambda$ theory will be dealt with in more detail in an upcoming publication [27].

As the last class we mention the warped compactifications. Here the non-Abelian gauge symmetry can appear in two ways. Either it arises from the bulk gauge fields somehow confined to the branes [32], or from the internal degrees of freedom on the branes, as in the Horava-Witten scenario [33] or its generalizations and variants. In either cases we face the instability discussed here and before using these setups for any model building the instability issue should be addressed.

\section{Concluding remarks}

In this paper we have studied the instability of general spherically symmetric solutions of four dimensional EYMD theories with a constant (magnetic) field strength on the sphere. We showed that the same mode which causes the instability in the gravitation cases is also 
present in the non-gravitational case. The instability is due to the fact that the lowest modes of gauge bosons in the background of a Dirac magnetic monopole on the flat space-time is "tachyonic" and has an exponential growth in time.

We showed that a Dirac magnetic monopole of a generic charge in a non-Abelian gauge theory causes instability in the fluctuations of the gauge fields. We should stress that, as discussed in section 2.1, although this statement is true for a generic charge there exists cases corresponding to the minimum allowed value of charges that there are no tachyons and are hence stable. For example, for the $S U(2)$ gauge group, that is $n=1$. As discussed in section 3 , these cases remain stable when gravity is turned on.

One may wonder about the dynamics of the tachyon and ask for a tachyon condensation mechanism with or without gravity. One should note that the tachyon is a feature of the linearized analysis. Its exponential growth will eventually make the linearized analysis invalid. A tachyon condensation of some kind may be the final fate of this instability. However, for this an independent analysis is called for.

An interesting outcome of our main result, which was indeed our original motivation for looking into this problem, concerns the attractor mechanism [9, 10] in extremal (supersymmetric or non-supersymmetric) blackholes. These are charged blackhole solutions to Einstein-Maxwell-Dilaton theory. According to the attractor mechanism the near horizon behavior of the blackhole solution is independent of the value of the dilatonic fields and only depends on the charges defining the solution. In the case of the colored black holes, due to the instability discussed here, the straightforward application of the attractor mechanism becomes questionable.

The instability of the colored blackholes can also be relevant to resolving the moduli problem in string compactification and removing some of the pieces of the string landscape [34]. In a sense this instability could be used as another criterium for distinguishing the swampland from the landscape [35].

Here we mainly focused on the EYM theory in four dimensions. One of the cases which is of outmost interest is the EYM- $\Lambda$ theories. As briefly discussed in section 4.2 these theories are very relevant to string theory. It is interesting to study the (in)stability of the embedded Abelian, colored blackhole solutions in the context of $4 d$ gauged supergravities [27]. Among this class the case of $4 d S O(8)$ gauged supergravity, which is related to M-theory on $S^{7}$ and the stability of the $4 d$ AdS-RN blackholes are of particular interest. It will also be very interesting to generalize our analysis of section 3 to the cases in higher dimensions and check whether the tachyonic modes still persist. We would expect that the kind of instability present in four dimensions should also appear in higher dimensions. This expectation should, however, be checked by explicit computations. One of the most interesting cases concerns the 
charged blackhole configurations of the $5 d S U(4)$ gauged supergravity, arising from $I I B / S^{5}$. These are the cases relevant to the AdS/CFT duality [27].

\section{Acknowledgements}

We are grateful to Gary Gibbons for bringing some old references on the subject of colored black holes and their instabilities to our attention. We would like to express our gratitude to the organizers of the Third Crete Regional Conference where this project was initiated. A.E.M would also like to thank the high energy section of the Abdus-Salam ICTP where some of this research was carried out.

\section{References}

[1] A. Sen, "Black hole solutions in heterotic string theory on a torus," Nucl. Phys. B 440, 421 (1995) arXiv:hep-th/9411187.

[2] R. Bartnik and J. Mckinnon, "Particle-Like Solutions Of The Einstein Yang-Mills Equations," Phys. Rev. Lett. 61, 141 (1988).

J. A. Smoller and A. G. Wasserman, "Existence of infinitely many smooth, static, global solutions of the Einstein / Yang-Mills equations," Commun. Math. Phys. 151, 303 (1993).

[3] P. Van Nieuwenhuizen, D. Wilkinson and M. J. Perry, "On A Regular Solution Of 'T Hooft's Magnetic Monopole Model In Curved Space," Phys. Rev. D 13, 778 (1976). M. J. Perry, "Black Holes Are Colored," Phys. Lett. B 71 (1977) 234.

[4] J. A. Smoller, A. G. Wasserman and S. T. Yau, "Existence of black hole solutions for the Einstein / Yang-Mills equations," Commun. Math. Phys. 154, 377 (1993).

P. Breitenlohner, P. Forgacs and D. Maison, "On Static spherically symmetric solutions of the Einstein Yang-Mills equations," Commun. Math. Phys. 163, 141 (1994).

[5] G. V. Lavrelashvili and D. Maison, "Regular and black hole solutions of Einstein YangMills Dilaton theory," Nucl. Phys. B 410, 407 (1993).

[6] M. S. Volkov and D. V. Gal'tsov, "Gravitating non-Abelian solitons and black holes with Yang-Mills fields," Phys. Rept. 319, 1 (1999) arXiv:hep-th/9810070.

[7] S. Randjbar-Daemi, A. Salam and J. A. Strathdee, "Instability Of Higher Dimensional Yang-Mills Systems," Phys. Lett. B 124, 345 (1983) [Erratum-ibid. B 144, 455 (1984)]. 
[8] G. R. Dvali, S. Randjbar-Daemi and R. Tabbash, "The origin of spontaneous symmetry breaking in theories with large extra dimensions," Phys. Rev. D 65, 064021 (2002) arXiv:hep-ph/0102307.

[9] S. Ferrara and R. Kallosh, "Supersymmetry and Attractors," Phys. Rev. D 54, 1514 (1996) arXiv:hep-th/9602136.

[10] K. Goldstein, N. Iizuka, R. P. Jena and S. P. Trivedi, "Non-supersymmetric attractors," Phys. Rev. D 72, 124021 (2005), arXiv:hep-th/0507096.

[11] L. Alvarez-Gaume and S. F. Hassan, "Introduction to S-duality in N = 2 supersymmetric gauge theories: A pedagogical review of the work of Seiberg and Witten," Fortsch. Phys. 45, 159 (1997) arXiv:hep-th/9701069.

[12] K. M. Lee, V. P. Nair and E. J. Weinberg, "A Classical instability of Reissner-Nordström solutions and the fate of magnetically charged black holes," Phys. Rev. Lett. 68, 1100 (1992) arXiv:hep-th/9111045.

[13] A. A. Ershov and D. V. Galtsov, "Nonexistence Of Regular Monopoles And Dyons In The SU(2) Einstein Yang-Mills Theory," Phys. Lett. A 150, 159 (1990).

[14] S. Randjbar-Daemi, A. Salam and J. A. Strathdee, "Spontaneous Compactification In Six-Dimensional Einstein-Maxwell Theory," Nucl. Phys. B 214, 491 (1983).

[15] D. Lohiya, "Stability Of Einstein Yang-Mills Monopoles And Dyons," Annals Phys. 141, 104 (1982).

[16] M. E. Ortiz, "Curved space magnetic monopoles," Phys. Rev. D 45, 2586 (1992).

P. Breitenlohner, P. Forgacs and D. Maison, "Gravitating monopole solutions 1\& 2," Nucl. Phys. B 383, 357 (1992); Nucl. Phys. B 442, 126 (1995) arXiv:gr-qc/9412039.

[17] P. Forgacs and S. Reuillon, "Spatially compact solutions and stabilization in EinsteinYang-Mills-Higgs theories," Phys. Rev. Lett. 95, 061101 (2005) [arXiv:gr-qc/0505007].

[18] W. H. Aschbacher, "On the instabilities of the static, spherically symmetric SU(2) Einstein-Yang-Mills-dilaton solitons and black holes," Phys. Rev. D 73, 024014 (2006) arXiv:gr-qc/0509060].

[19] T. Torii, K. i. Maeda and T. Tachizawa, "Cosmic colored black holes," Phys. Rev. D 52, 4272 (1995) arXiv:gr-qc/9506018. 
M. S. Volkov, N. Straumann, G. V. Lavrelashvili, M. Heusler and O. Brodbeck, "Cosmological Analogues of the Bartnik-McKinnon Solutions," Phys. Rev. D 54, 7243 (1996) arXiv:hep-th/9605089.

[20] J. Bjoraker and Y. Hosotani, "Stable monopole and dyon solutions in the Einstein-YangMills theory in asymptotically anti-de Sitter space," Phys. Rev. Lett. 84, 1853 (2000) arXiv:gr-qc/9906091; "Monopoles, dyons and black holes in the four-dimensional Einstein-Yang-Mills theory," Phys. Rev. D 62, 043513 (2000) arXiv:hep-th/0002098.

E. Winstanley, "Existence of stable hairy black holes in SU(2) Einstein-Yang-Mills theory with a negative cosmological constant," Class. Quant. Grav. 16, 1963 (1999) arXiv:gr-qc/9812064.

[21] P. Breitenlohner, D. Maison and G. Lavrelashvili, "Non-Abelian gravitating solitons with negative cosmological constant," Class. Quant. Grav. 21, 1667 (2004) [arXiv:gr-qc/0307029].

P. Breitenlohner, P. Forgacs and D. Maison, "Classification of static, spherically symmetric solutions of the Einstein-Yang-Mills theory with positive cosmological constant," Commun. Math. Phys. 261, 569 (2006) arXiv:gr-qc/0412067].

P. Forgacs and S. Reuillon, "On the number of instabilities of cosmological solutions in an Einstein-Yang-Mills system," Phys. Lett. B 568, 291 (2003) arXiv:hep-th/0306126.

[22] Landau Text book Series, Quantum Mechanics, page 73, problem 5.

[23] R. Kallosh, N. Sivanandam and M. Soroush, "The non-BPS black hole attractor equation," JHEP 0603, 060 (2006) arXiv:hep-th/0602005.

[24] K. Goldstein, R. P. Jena, G. Mandal and S. P. Trivedi, "A C-function for nonsupersymmetric attractors," JHEP 0602, 053 (2006) arXiv:hep-th/0512138.

P. Kaura and A. Misra, "On the existence of non-supersymmetric black hole attractors for two-parameter Calabi-Yau's and attractor equations," arXiv:hep-th/0607132.

[25] J. M. Maldacena, J. Michelson and A. Strominger, "Anti-de Sitter fragmentation," JHEP 9902, 011 (1999) arXiv:hep-th/9812073.

[26] P. Breitenlohner and D. Z. Freedman, "Positive Energy In Anti-De Sitter Backgrounds And Gauged Extended Supergravity," Phys. Lett. B 115, 197 (1982).

E. Witten, "Anti-de Sitter space and holography," Adv. Theor. Math. Phys. 2, 253 (1998) arXiv:hep-th/9802150. 
[27] A. E. Mosaffa, S. Randjbar-Daemi, M.M. Sheikh-Jabbari, "Instabilities in Magnetized Four and Five Dimensional Gauged Supergravity Solutions", Work in Progress.

[28] A. Sen, "Black hole entropy function and the attractor mechanism in higher derivative gravity," JHEP 0509, 038 (2005) arXiv:hep-th/0506177].

[29] A. Dabholkar, A. Sen and S. Trivedi, "Black Hole Microstates and Attractor Without Supersymmetry," arXiv:hep-th/0611143.

D. Astefanesei, K. Goldstein and S. Mahapatra, "Moduli and (un)attractor black hole thermodynamics," arXiv:hep-th/0611140.

[30] J. Polchinski, "String Theory", Vol.1 \& 2, Cambridge University Press, 1997.

[31] J. G. Russo and A. A. Tseytlin, "Constant magnetic field in closed string theory: An Exactly solvable model," Nucl. Phys. B 448, 293 (1995) arXiv:hep-th/9411099].

[32] S. Parameswaran, S. Randjbar-Daemi and A. Salvio "Guage fields, fermions and mass gaps in 6D brane words," arXiv:hep-th/0608074.

[33] P. Horava and E. Witten, "Heterotic and type I string dynamics from eleven dimensions," Nucl. Phys. B 460, 506 (1996) arXiv:hep-th/9510209]; "Eleven-Dimensional Supergravity on a Manifold with Boundary," Nucl. Phys. B 475, 94 (1996) arXiv:hep-th/9603142.

[34] L. Susskind, "The anthropic landscape of string theory," arXiv:hep-th/0302219.

[35] C. Vafa, "The string landscape and the swampland," arXiv:hep-th/0509212. H. Ooguri and C. Vafa, "On the geometry of the string landscape and the swampland," arXiv:hep-th/0605264. 